\title{
Ganancia de peso y rendimiento de carcasa de llamas (Lama glama) dientes de leche sometidas a engorde con cuatro tipos de alimentación
}

\author{
Body weight gain and carcass yield of llamas (Lama glama) subjected to \\ fattening under four feeding types
}

Ricardo Robles ${ }^{1}$, Víctor Hidalgo ${ }^{2,4}$, María Wurzinger ${ }^{3}$, Gustavo Gutierrez ${ }^{2}$

\section{Resumen}

\begin{abstract}
El objetivo del estudio fue evaluar la ganancia de peso y rendimiento de carcasa de llamas machos diente de leche sometidas a engorde con cuatro tipos de alimentación (T1: Pastura natural; T2: T1 + dosificación vitamínica; T3: T1 + heno de alfalfa; T4: T2 + heno de alfalfa). Se utilizó un diseño completamente al azar con arreglo factorial de $2 \times 2$ con un análisis de covarianza (peso vivo inicial como covariable). Se emplearon 32 llamas asignadas al azar a uno de los cuatro tratamientos por 84 días. La carga animal empleada fue de 1.3 unidades llama/ha. La ganancia diaria de peso (GDP) se evaluó en los periodos de engorde a 14, 28, 42, 56, 70 y 84 días. La GDP por periodo de engorde fue significativamente diferente entre periodos, donde los primeros tres periodos (14, 28 y 42 días) mostraron mejor GDP (337, 237 y $160 \mathrm{~g} / \mathrm{d}$, respectivamente). La GDP por tipo de alimentación fue mayor en T3 $(157 \mathrm{~g} / \mathrm{d})$ durante el periodo de 84 días $(\mathrm{p}<0.01)$ y el mejor rendimiento de carcasa se obtuvo en T4 (53.60\%) seguido por el T3 (53.20\%) $(\mathrm{p}<0.01)$. Se concluye que la mayor GDP se logra hasta los 42 días y el mejor rendimiento de carcasa se logra en la pastura natural + suplementación con heno de alfalfa (T3).
\end{abstract}

Palabra clave: rendimiento de carcasa, engorde, llamas

\section{Abstract}

The aim of this study was to evaluate the bodyweight gain and carcass yield of young male llamas (milk tooth) subjected to fattening with four types of feeding (T1: Natural pasture; T2: T1 + vitamin injection; T3: T1 + alfalfa hay; T4: T2 + hay alfalfa). A

\footnotetext{
${ }^{1}$ Universidad Nacional Daniel Alcides Carrión de Cerro de Pasco, Perú

${ }^{2}$ Universidad Nacional Agraria La Molina, Lima, Perú

${ }^{3}$ BOKU - University of Natural Resources and Life Sciences, Vienna, Austria

${ }^{4}$ E-mail: vhidalgo@lamolina.edu.pe
}

Recibido: 29 de marzo de 2019

Aceptado para publicación: 23 de diciembre de 2019

Punlicado: 31 de marzo de 2020 
completely randomized design with a $2 \times 2$ factorial arrangement with a covariance analysis (initial live weight as covariate) was used. Thirty-two llamas were randomized assigned to one of the four treatments for 84 days. The stocking rate was 1.3 animal units/ha. Daily weight gain (DWG) was evaluated in the fattening periods at 14, 28, 42, 56, 70 and 84 days. The DWG per fattening period was significantly different between periods, in the first three periods (14, 28 and 42 days) showing the best DWG $(337,237$ and $160 \mathrm{~g} / \mathrm{d}$, respectively). The DWG per feed type was higher in T3 $(157 \mathrm{~g} / \mathrm{d})$ during the 84-day period $(\mathrm{p}<0.01)$ and the best carcass yield was obtained in T4 $(53.60 \%)$ followed by T3 $(53.20 \%)(p<0.01)$. It is concluded that the highest DWG is achieved at day 42 and the best carcass yield is obtained with natural pasture + alfalfa hay supplementation (T3).

Key words: carcass dressing percentage, fattening, llama

\section{INTRODUCCIÓN}

Los camélidos sudamericanos (CSA) constituyen un recurso genético de gran importancia social, económica, cultural y científica para el Perú y otros países de la Región Andina (FAO, 2005a). Existe una tendencia creciente de incentivos para la producción de carne de camélidos sudamericanos, especialmente de llama, debido que representa la más importante fuente de proteína de origen animal para la población andina (Pérez et al., 2000); sin embargo, la carne que se vende para el consumo humano son mayormente de adultos con alta incidencia de sarcocistosis (Castro et al., 2004) y de baja condición corporal, dado el tipo de alimentación basado en pasto natural de baja calidad. Por tanto, un adecuado manejo de la alimentación de estos animales podría aumentar la producción y los ingresos económicos del productor (Salvá, 2000).

Cristofanelli et al. (2004) indican que la llama, en las zonas altoandinas, es la más adecuada para producir carne; de allí la importancia de ampliar los estudios sobre engorde de esta especie. La llama, por otra parte, cumple una función muy importante como medio de transporte en los lugares carentes de una adecuada infraestructura vial (FAO, 2005b). Actualmente, el mercado de consumo es más exigente en calidad, palatabilidad y salubridad de la carne, requisitos que pue- den ser cubiertos con el sacrificio de animales jóvenes y bien alimentados (Cristofanelli et al., 2004).

Entre las particularidades anatómicas y fisiológicas de los CSA se encuentra su gran capacidad de adaptación a las condiciones de hipoxia y de escasez de recursos forrajeros en las alturas. Así, el mayor tiempo de retención del alimento en el tracto digestivo, que les permite una mayor fermentación microbiana de la fracción fibrosa del alimento (San Martín y Van Saun, 2014), pudiendo incluso tener un mejor aprovechamiento de pasturas de baja calidad (Genin et al., 1994). Con respecto al metabolismo del nitrógeno, no se necesita aumentar los niveles de proteína en la dieta para un eficiente reciclaje de nitrógeno (San Martín y Van Saun, 2014). Por otro lado, el metabolismo de la glucosa en los CSA es un enigma, conociéndose que su concentración es de $126 \mathrm{mg} / \mathrm{dl}$ de plasma en promedio, con rangos entre 103 y $160 \mathrm{mg} / \mathrm{dl}$, aunque se elevan a más de $200-300 \mathrm{mg} / \mathrm{dl}$ en condiciones mínimas de estrés (Araya et al., 2000; Cebra et al., 2001).

Es posible realizar el destete, tanto en llamas como en alpacas, a una temprana edad, si se dispone de estrategias de suplementación que permitan alcanzar el peso óptimo deseado (San Martín, 1996); así mismo, este autor menciona que es posible obtener carcasas de buena calidad mediante el engorde de animales jóvenes. Dentro de estas estrategias 
de alimentación, se tiene el uso de pastos cultivados, la suplementación energéticaproteica y las reservas de áreas de pastoreo con pastos de buena calidad. Se debe considerar que los pastos cultivados irrigados son económicamente beneficiosos si se usan como un suplemento para las praderas y no como una base alimenticia (Macuchapi, 2006).

Se estima que el porcentaje de saca anual, tanto en alpacas como en llamas, es alrededor de 10-12\% (FAO, 2005a), constituida mayormente por animales que han llegado al final de su vida productiva. Esto hace que la presencia de sarcocistosis en la musculatura sea elevada (Castro et al., 2004), y que la carne sea de baja calidad. Sin embargo, el cuarto posterior de la llama presenta los cortes de mayor valor y de mayor proporción de carne que la alpaca (Cristofanelli et al., 2005). Al respecto, Bustinza (2001), menciona que el rendimiento de carcasa es menor en animales de 2 años (56.2\%) y mayor en animales de 3 y 4 años (59.5\%). Cristofanelli et al. (2004), Mamani-Linares y Gallo (2013 a,b) reportan rendimientos de carcasa de 51 y $57 \%$, respectivamente, en llamas. Por otro lado, la carne de estas especies presenta un contenido proteico entre 21.5 y $23.9 \%$, bajos niveles de grasa $(0.49-2.05 \%)$ y de colesterol (39.0-56.3 mg/100 g) comparada con otras carnes rojas (Cristofanelli et al., 2004; Mamani-Linares y Gallo, 2013a).

El presente estudio tuvo por objetivo evaluar la respuesta productiva en ganancia de peso y rendimiento de carcasa de llamas machos diente de leche sometidas a engorde bajo cuatro tipos de alimentación.

\section{Materiales y Métodos}

El estudio se realizó en la Unidad de Producción Galaumarca, propiedad de la Comunidad Campesina San Pedro de Ninacaca, localizado a $4350 \mathrm{msnm}$, en el distrito de Ninacaca, provincia de Pasco, Perú.
Cuadro 1. Clasificación de la condición de la pastura natural para llamas en el área experimental (Inicio de la época seca)

\begin{tabular}{|c|c|}
\hline Indicadores & Puntaje \\
\hline $\begin{array}{l}\text { Composición de } \\
\text { especies } \\
\text { decrecientes }\end{array}$ & 71 \\
\hline Índice forrajero & 86 \\
\hline Cobertura & 93 \\
\hline Índice de vigor & 67 \\
\hline Total & 78 \\
\hline $\begin{array}{l}\text { Condición del } \\
\text { pastizal }^{1}\end{array}$ & Buena \\
\hline $\begin{array}{l}\text { Capacidad de carga } \\
\text { estimada }^{2}\end{array}$ & $\begin{array}{l}1.3 \text { unidades } \\
\text { llama/ha/año } \\
\text { (15.6 unidades } \\
\text { llama mes) }\end{array}$ \\
\hline $\begin{array}{l}\text { Fuente: }{ }^{1} \text { Laboratori } \\
\text { Pastizales (LUP), Unive } \\
\text { La Molina; }{ }^{2} \text { San Martin } \\
\text { y Malpartida (1992) }\end{array}$ & $\begin{array}{l}\text { de Utilización de } \\
\text { lad Nacional Agraria } \\
\text { Bryant (1989); Florez }\end{array}$ \\
\hline
\end{tabular}

La zona presenta un clima frígido o de tundra (4000 a $5000 \mathrm{msnm}$ ), con temperatura promedio anual de $4{ }^{\circ} \mathrm{C}\left(15^{\circ} \mathrm{C}\right.$ de día y menor de $0{ }^{\circ} \mathrm{C}$ por las noches), temperatura mínima promedio de $-11^{\circ} \mathrm{C}$, y precipitación anual más frecuente entre los meses de noviembre y marzo (650-900 mm).

Se establecieron cuatro tratamientos: T1: Pasto natural; T2: Pasto natural + vitaminas; T3: Pasto natural + suplemento con heno de alfalfa; T4: Pasto natural + suplemento con heno de alfalfa + vitaminas. La cantidad de heno suplementado por día fue el $30 \%$ del consumo de materia seca esperado (Van Saun, 2006). El suplemento de vitaminas A, D, E (Vigantol) y B12 (Catosal), ambas del Laboratorio Bayer, fueron inyectadas en dosis de 2 y $5 \mathrm{ml}$ por animal, respectivamente, vía i.m.. El uso de vitaminas se justifica por considerarse que la pastura es deficiente, especialmente en la época seca y en el heno de alfalfa (San Martin y Van Saun, 2014). 
Se realizó un censo de la vegetación del campo para determinar la condición del pastizal, evaluándose los siguientes indicadores: (a) especies decrecientes o deseables (palatables) y que producen abundante forraje, (b) el índice forrajero (suma de estas especies más aquellas poco deseables), (c) la cobertura (suma de las especies deseables + especies poco deseable + especies indeseables), (d) índice de vigor de las plantas nativas (tamaño, diámetro de base y proporción de sus macollos y florales), de acuerdo a Florez y Malpartida (1992) (ver Cuadro 1).

Las muestras de pasto fueron tomadas del área del ensayo al azar al inicio del periodo experimental, considerando 15 muestras de $0.5 \mathrm{~m}^{2}$ cada una. Estas fueron mezcladas y se tomó una muestra mixta representativa que fue enviada al Laboratorio de Evaluación Nutricional de Alimentos de la Facultad de Zootecnia, Universidad Nacional Agraria La Molina, para el análisis químico proximal, según el método de la AOAC (2005). La composición botánica de la pradera mostró una alta heterogeneidad de especies, predominando Festuca humilior (30\%), Calamagrostis vicunarum (28\%) y Carex sp (13\%). En el Cuadro 1 se presenta la clasificación por tipo de condición, obtenida a partir de los censos de vegetación por el método de Parker al inicio de la prueba experimental y durante el inicio de la época seca. La pastura era de condición buena para llamas, estimando una capacidad de carga de 1.30 unidades 1la$\mathrm{ma} / \mathrm{ha} / \mathrm{año} \mathrm{(15.6} \mathrm{unidades} \mathrm{por} \mathrm{mes).}$

Se utilizaron 32 llamas machos de 16 a 20 meses (determinado mediante cronología dentaria), de genotipo heterogéneo, procedentes de varias comunidades campesinas, con peso promedio de $56 \mathrm{~kg}$. Los animales fueron distribuidos al azar en los cuatro tratamientos. El periodo preexperimental se inició en abril de 2016 (inicio de la época seca), la fase de adaptación tuvo una duración de tres semanas, donde se realizaron los tratamientos sanitarios preventivos de despara-sitación interna y externa (Ivermectina).
En el periodo experimental, todos los animales ocuparon el mismo campo de pastoreo, de 5.5 ha durante 84 días, equivalente a una carga animal de 15.6 unidades llama mes (Cuadro 1). El área de pastura natural utilizado fue cercada con alambre de púas con tendido de 6 hilos utilizando postes de eucalipto de $10 \mathrm{~cm}$ de diámetro y $2.20 \mathrm{~m}$ de altura. Los animales permanecieron en el campo de pastoreo por 9 horas por día (08:0017:00). Los animales fueron conducidos en las tardes al cobertizo de la comunidad, donde se acondicionó un corral de $40 \mathrm{~m}^{2}$ para 16 animales (T1 y T2) y 16 corrales individuales de $4.5 \mathrm{~m}^{2} /$ animal para los tratamientos T3 y T4 (ocho llamas por tratamiento). El suplemento de heno de alfalfa se suministró por corrales en forma diaria a las 17:00.

Se evaluaron seis periodos con intervalos de 14 días (0-14, 15-28, 29-42, 43-56, 5770, 71-84 días) registrando el peso vivo y determinando la ganancia de peso diaria. Del mismo modo, se consideró un periodo de evaluación general de 0 a 84 días, registrando el peso vivo inicial y peso vivo final y calculando la ganancia de peso por tratamiento (tipo de alimentación).

\section{Se evaluaron los siguientes parámetros:}

- Peso vivo: Se midió utilizando una balanza digital electrónica de plataforma (sistema con Barras Nacionales de Carga Modelo BR-2000, $2000 \mathrm{~kg}$ x $1 \mathrm{~kg}$, sistema de barras Modelo: BR4000 e indicador digital de peso modelo XK315A de precisión $1 \mathrm{~kg}$ ).

- La ganancia de peso diario y por periodo de engorde se calculó con el peso inicial por periodo y para todo el periodo experimental.

- Rendimiento de carcasa: Expresado en porcentaje. Se determinó por la relación entre el peso de la carcasa (caliente y frío) y el peso vivo al sacrificio.

Los datos productivos fueron analizados por un Diseño Completamente Aleatorizado con arreglo factorial $2 \times 2$, con- 
Cuadro 2. Análisis químico proximal del pasto natural y del heno de alfalfa, al inicio del periodo del experimento (base seca) (Ninacaca, Pasco, Perú)

\begin{tabular}{lcc}
\hline & $\begin{array}{c}\text { Pasto } \\
\text { natural }\end{array}$ & $\begin{array}{c}\text { Heno de } \\
\text { alfalfa }\end{array}$ \\
\hline Materia seca (\%) & 91.38 & 89.23 \\
Proteína cruda (\%) & 8.19 & 17.90 \\
Grasa (\%) & 1.82 & 1.95 \\
Fibra cruda (\%) & 31.36 & 27.80 \\
Ceniza (\%) & 3.49 & 8.12 \\
ELN (\%) & 55.14 & 44.27 \\
\hline
\end{tabular}

Fuente: Laboratorio de Evaluación Nutricional de Alimentos, Universidad Nacional Agraria La Molina

siderándose el peso vivo inicial como covariable. Los factores fueron: tipo de suplementación (con y sin suplementación) y dosificación (con y sin dosificación vitamínica). Las diferencias entre tratamientos se determinaron mediante la prueba de diferencias de límite de significación (DLS). Así mismo, los resultados de ganancia de peso diario y por periodos de engorde se analizaron mediante un modelo estadístico longitudinal y las diferencias entre periodos de engorde se determinaron mediante la prueba de DLS, utilizando un nivel de confianza de $99 \%$.

\section{Resultados y Discusión}

El pasto y el heno de alfalfa tienen diferente contenido de nutrientes, especialmente en proteína cruda (PC) (Cuadro 2). Según la literatura, los niveles proteicos de las dietas (pradera natural) para las llamas están entre $1.4 \%$ de PC en una pradera de Festuca orthophylla (Genin et al., 1994) hasta 7.1\% en una pradera de Festuca dolichophylla Calamagrostis vicunarum (Flores, 2006) y desde $11.2 \%$ en una asociación Dactylis glomerata - Trifolium repens L (Flores,
2006). El heno de alfalfa puede aportar hasta 19.4\% de PC en base seca (López et al., 2000).

Flores (2006) indica que la pradera natural Festuca humilior - Calamagrostis vicunarum utilizado en el pastoreo de los animales presenta niveles de $8.2 \%$ de PC, la cual se encontraría por encima de los límites inferiores críticos $(7.0 \%)$ considerados para mantenimiento. Por lo tanto, el aporte de nutrientes de los pastos naturales ( $\mathrm{T} 1$ y $\mathrm{T} 2$ ) sería insuficiente en términos generales para satisfacer los requerimientos de crecimiento de las llamas en la estación seca.

Las llamas alimentadas con pastura natural y suplementadas con heno de alfalfa (T3) mostraron un valor promedio más alto para el peso vivo final que los animales de los otros tratamientos $(\mathrm{p}<0.01)$, mientras que T3 y T4 fueron similares y tuvieron mejor ganancia diaria de peso y porcentaje de ganancia de peso que T1 y T2 $(\mathrm{p}<0.01)$. Por otro lado, los valores productivos evaluados de $\mathrm{T} 1$ y $\mathrm{T} 2$ fueron similares (Cuadro 3).

Las diferencias en el peso vivo final estarían relacionadas con el mayor aporte de nutrientes por parte del heno de alfalfa (Van Saun, 2006). Al respecto, Mamani-Linares y Gallo (2012) reportaron ganancias de $105 \mathrm{~g} / \mathrm{d}$ en llamas al pastoreo en pradera nativa entre los meses de setiembre y noviembre con $4.8 \%$ de PC y con predominancia de Festuca dolichophylla, Stipa ichu, Muhlenbergia spp, Bromus unioloides, Calamagrotis spp y Festuca orthophylla. Dichos valores son similares a los obtenidos en este estudio (106 g/d).

Así mismo, la mayor ganancia de peso $(157 \mathrm{~g} / \mathrm{d})$ en $\mathrm{T} 3$ con solo suplemento de heno de alfalfa (Cuadro 3) se explica igualmente por el mayor aporte de nutrientes de la alfalfa (Cuadro 2), que permitió cubrir los requerimientos de mantenimiento y ganancia de peso. Al respecto, García et al. (2002) en un estudio con llamas al pastoreo utilizando Lolium perenne + Trifolium repens $\mathrm{L}$ y 
Cuadro 3. Peso vivo y ganancia de peso diario por tratamiento en llamas dientes de leche sometidas a cuatro tipos de alimentación en praderas naturales (Ninacaca, Pasco, Perú)

\begin{tabular}{cccccc}
\hline Tratamientos $^{1}$ & $\begin{array}{c}\text { Peso vivo } \\
\text { inicial } \\
(\mathrm{kg})\end{array}$ & $\begin{array}{c}\text { Peso vivo } \\
\text { final } \\
(\mathrm{kg})\end{array}$ & $\begin{array}{c}\text { Ganancia de } \\
\text { peso total } \\
(\mathrm{kg})\end{array}$ & $\begin{array}{c}\text { Ganancia diaria } \\
\text { de peso } \\
(\mathrm{kg})\end{array}$ & $\begin{array}{c}\text { Ganancia de } \\
\text { peso } \\
(\%)\end{array}$ \\
\hline $\mathrm{T} 1$ & $55.24^{\mathrm{a}}$ & $64.24^{\mathrm{b}}$ & $9.00^{\mathrm{b}}$ & $0.106^{\mathrm{b}}$ & $16.29^{\mathrm{ab}}$ \\
$\mathrm{T} 2$ & $57.55^{\mathrm{a}}$ & $66.30^{\mathrm{b}}$ & $8.75^{\mathrm{b}}$ & $0.103^{\mathrm{b}}$ & $15.20^{\mathrm{b}}$ \\
$\mathrm{T} 3$ & $57.53^{\mathrm{a}}$ & $70.88^{\mathrm{a}}$ & $13.35^{\mathrm{a}}$ & $0.157^{\mathrm{a}}$ & $23.21^{\mathrm{a}}$ \\
$\mathrm{T} 4$ & $54.06^{\mathrm{a}}$ & $65.11^{\mathrm{b}}$ & $11.05^{\mathrm{ab}}$ & $0.131^{\mathrm{ab}}$ & $20.44^{\mathrm{ab}}$ \\
\hline
\end{tabular}

$a, b$ Letras diferentes en columnas indican diferencias significativas $(p<0.01)$

${ }^{1}$ T1: Pasto natural; T2: Pasto natural + vitaminas; T3: Pasto natural + heno de alfalfa; T4: Pasto natural + heno de alfalfa + vitaminas

Phalaris arundinacea + Trifolium repens $\mathrm{L}$ reportaron ganancias de peso de 199 y $182 \mathrm{~g} / \mathrm{d}$, respectivamente, superiores a lo obtenido en el presente estudio, posiblemente debido al empleo de pastos cultivados como dieta única. No obstante, los pastos cultivados irrigados son económicamente beneficiosos si se usan como un suplemento para las praderas nativas y no como una base alimenticia por su mayor costo (San Martín y Van Saun, 2014).

Las ganancias de peso entre tratamientos fueron diferentes entre los tres primeros periodos de engorde (14, 28 y 42 días) en comparación con los otros periodos. Estas respuestas concuerdan con valores obtenidos en llamas y alpacas (Turín et al., 1999; García et al., 2002), debido a la mayor disponibilidad y calidad de pasto de las praderas naturales al inicio de la prueba experimental (Florez y Malpartida, 1992; San Martín, 1996). Este periodo coincidió con el final de la época de lluvias (marzo-abril), donde el contenido de proteína cruda era de $8.19 \%$ en base seca. Por otro lado, la menor ganancia de peso se presentó entre el periodo 43-56 y el periodo 57-70 días, que comprendió los meses de junio y julio, donde la época seca se acentúa con mayor intensidad. Los resultados de ganancia de peso, no obstante, fueron superiores a los obtenidos por García et al. (2002) y Macuchapi (2006).
Las llamas utilizadas en el presente estudio fueron adquiridas de pequeños productores, donde el manejo de la tierra es de tipo comunal, con frecuencia con una fuerte tendencia al sobrepastoreo, lo que va en detrimento de una producción sostenible (FAO 2005a). Entonces, esta diferencia en la respuesta animal por periodos de engorde (Cuadro 4) puede ser explicada por el crecimiento compensatorio que usualmente se observa en los periodos iniciales del engorde (García et al., 2002).

El peso de la carcasa caliente y el peso de la carcasa fría fue significativamente superior en las llamas de T3 (suplementación con heno de alfalfa) $(\mathrm{p}<0.01)$, no habiendo diferencias entre los demás tratamientos (Cuadro 5). Estos valores son superiores a los reportados por Mamani-Linares y Gallo (2013b), quienes encontraron valores para llamas de la misma edad de $23.8,24.8$ y $29.7 \mathrm{~kg}$ como peso promedio de carcasa caliente y $23.0,23.8$ y $28.6 \mathrm{~kg}$ como peso promedio de la carcasa fría para las dietas con pasto natural (GR), GR + heno y GR + concentrado, respectivamente. Sin embargo, los valores del rendimiento de carcasa fría fueron similares a los reportados por Cristofanelli et al. (2004), Arzabe (2007) y Laura (2012), pero superiores al valor reportado por Mamani-Linares y Gallo (2013b) de 48.3\%. Estas diferencias 
Cuadro 4. Ganancia de peso (g/día) en llamas dientes de leche bajo cuatro tipos de alimentación en praderas naturales en diferentes periodos de engorde (Ninacaca, Pasco, Perú)

\begin{tabular}{cccccccc}
\hline Trat. & \multicolumn{7}{c}{ Periodos (días) } \\
\cline { 2 - 8 } & $(0-14)$ & $(15-28)$ & $(29-42)$ & $(43-56)$ & $(57-70)$ & $(71-84)$ & $(0-84)$ \\
\hline T 1 & $270^{\mathrm{ab}}$ & $194^{\mathrm{b}}$ & $118^{\mathrm{b}}$ & $7^{\mathrm{a}}$ & $20^{\mathrm{b}}$ & $33^{\mathrm{c}}$ & $107^{\mathrm{b}}$ \\
T 2 & $250^{\mathrm{b}}$ & $075^{\mathrm{c}}$ & $138^{\mathrm{ab}}$ & $-40^{\mathrm{b}}$ & $59^{\mathrm{b}}$ & $142^{\mathrm{a}}$ & $103^{\mathrm{b}}$ \\
T 3 & $334^{\mathrm{a}}$ & $237^{\mathrm{a}}$ & $138^{\mathrm{ab}}$ & $29^{\mathrm{a}}$ & $120^{\mathrm{a}}$ & $105^{\mathrm{b}}$ & $157^{\mathrm{a}}$ \\
T 4 & $337^{\mathrm{a}}$ & $183^{\mathrm{b}}$ & $160^{\mathrm{a}}$ & $-15^{\mathrm{b}}$ & $101^{\mathrm{a}}$ & $23^{\mathrm{c}}$ & $131^{\mathrm{ab}}$ \\
\hline
\end{tabular}

$a, b, c$ Letras diferentes en columnas indican diferencias significativas $(p<0.01)$

${ }^{1}$ T1: Pasto natural; T2: Pasto natural + vitaminas; T3: Pasto natural + heno de alfalfa; T4: Pasto natural + heno de alfalfa + vitaminas

Cuadro 5. Peso vivo final y rendimiento de carcasa de llamas diente de leche sometidas a engorde con cuatro tipos de alimentación sobre praderas naturales (Ninacaca, Pasco, Perú)

\begin{tabular}{lcccc}
\hline & T1 & T2 & T3 & T4 \\
\cline { 2 - 5 } & Prom. \pm DE & Prom. \pm DE & Prom. \pm DE & Prom. \pm DE \\
\hline Peso final (kg) & $64.24^{\mathrm{b}} \pm 9.37$ & $66.30^{\mathrm{b}} \pm 16.39$ & $70.88^{\mathrm{a}} \pm 15.09$ & $65.11^{\mathrm{b}} \pm 15.61$ \\
PCC (kg) & $34.46^{\mathrm{b}} \pm 5.51$ & $34.84^{\mathrm{b}} \pm 09.85$ & $37.63^{\mathrm{a}} \pm 7.94$ & $34.89^{\mathrm{b}} \pm 8.66$ \\
PCF (kg) & $33.41^{\mathrm{b}} \pm 5.44$ & $33.33^{\mathrm{b}} \pm 10.26$ & $36.51^{\mathrm{a}} \pm 8.07$ & $33.86^{\mathrm{b}} \pm 8.61$ \\
RCC (\%) & $53.60^{\mathrm{a}} \pm 3.50$ & $52.20^{\mathrm{a}} \pm 2.40$ & $53.20^{\mathrm{a}} \pm 2.40$ & $53.60^{\mathrm{a}} \pm 3.10$ \\
RCF (\%) & $52.00^{\mathrm{a}} \pm 2.90$ & $49.70^{\mathrm{a}} \pm 3.40$ & $51.50^{\mathrm{a}} \pm 2.20$ & $51.90^{\mathrm{a}} \pm 3.20$ \\
\hline
\end{tabular}

PCC: Peso carcasa caliente; PCF: Peso carcasa fría; RCC: Rendimiento carcasa caliente; RCF: Rendimiento carcasa fría

$a, b, c$ Letras diferentes en filas indican diferencias significativas $(p<0.01)$

${ }^{1}$ T1: Pasto natural; T2: Pasto natural + vitaminas; T3: Pasto natural + heno de alfalfa; T4: Pasto natural + heno de alfalfa + vitaminas

entre trabajos podrían deberse a diferencias en edades y tipos de animales, condición corporal y época del año (Mamani-Linares et al., 2014).

\section{Conclusiones}

- Las llamas alimentadas con pastura natural y suplementadas con heno de alfalfa tuvieron mayor ganancia de peso vivo $(\mathrm{p}<0.01)$.
- El mayor peso de carcasa caliente y frío $(p<0.01)$ se registró en llamas suplementadas con solo heno de alfalfa.

- La suplementación vitamínica no mejoró significativamente el comportamiento productivo durante el periodo de engorde de las llamas.

\section{Agradecimientos}

El presente trabajo fue financiado por el Programa Nacional de Innovación para la Competitividad y Productividad (Innóvate 
Perú), de acuerdo con el Contrato N. ${ }^{\circ} 129$ PNICP-PIAP-2015 «Mejoramiento de la producción, calidad y procesamiento tecnológico de la carne de llama procedente de la Sierra Central del Perú».

\section{Literatura Citada}

1. [AOAC] Association of Official Analytical Chemists. 2005. Official methods of analysis of the Association Agricultural Chemists. $18^{\text {th }}$ ed. Gaithersburg, Maryland, USA.

2. Araya AV, Atwater I, Navia MA, Jeffs S. 2000. Evaluation of insulin resistance in two kinds of South American camelids: llamas and alpacas. Comparative Med 50: 490-494.

3. Arzabe C.2007. Determinación del rendimiento y la rentabilidad de los cortes menores de la carne de llama (Lama glama L). Tesis de Ingeniero Agrónomo. La Paz, Bolivia: Univ. Mayor de San Andrés. 95 p.

4. Bustinza V. 2001. La alpaca. Puno, Perú: Univ. Nacional del Altiplano. $495 \mathrm{p}$.

5. Castro E, Sam R, López T, González A, Silva M. 2004. Evaluación de la edad como factor de riesgo de seropositividad a Sarcocystis sp en alpacas. Rev Inv Vet Perú 15: 83- 86. doi: 10.15381/ rivep.v15i1.1568

6. Cebra CK, Tornquist SJ, Van Saun RJ, Smith BB. 2001. Glucose tolerance testing in llamas and alpacas. Am J Vet Res 62: 682-686. doi: 10.2460/ ajvr.2001.62.682

7. Cristofanell S, Antonini A, Torres D, Polidori P, Renieri C. 2004. Meat and carcass quality from Peruvian llama (Lama glama) and alpaca (Lama pacos). Meat Sci 66: 589-593. doi: 10.1016/S0309-1740(03)00174-8

8. Cristofanelli S, Antonini A, Torres D, Polidori P, Renieri C. 2005. Carcass characteristics of Peruvian llama (Lama glama) and alpaca (Lama pacos) reared in the Andean highlands. Small Ruminant Res 58: 219-222. doi: 10.1016/j.smallrumres.2004.10.004

9. [FAO] Organización de las Naciones Unidas para la Agricultura y la Alimentación. 2005a. Situación actual de los camélidos sudamericanos en Bolivia. TCP/RLA/2914. Roma, Italia: FAO. 56 p.

10. [FAO] Organización de las Naciones Unidas para la Agricultura y la Alimentación. 2005b. Situación actual de los camélidos sudamericanos en Perú. TCP/ RLA/2914. Roma-Italia: FAO. 62 p.

11. Florez A, Malpartida E. 1992. Manejo de praderas nativas y pasturas en la región altoandina del Perú. Tomo I. Lima, Perú: Banco Agrario. 651 p.

12. Flores D. 2006. Producción primaria y flujo de energía en praderas naturales de Festuca-Calamagrostis y cultivados de Dactylis-trébol rojo. Tesis de Maestría. Lima, Perú: Univ. Nacional Agraria La Molina. $63 \mathrm{p}$.

13. García W, San Martín F, Novoa $C$, Franco E. 2002. Engorde de llamas bajo diferentes regímenes alimenticios. Rev Inv Vet Perú 13: 1-9. doi: 10.15381/ rivep.v13i2.7246

14. Genin D, Villca Z, Abasto P. 1994. Diet selection and utilization by llama and sheep in a high altitude-arid rangeland in Bolivia. J Range Manage 47: 245-248. doi: 10.2307/4003025

15. Laura L. 2012. Alternativas de agregación de valor con la transformación de productos derivados de carne de llama (Lama glama) en la localidad de Curahuara de Carangas, Oruro. Tesis de Ingeniero Agrónomo. La Paz, Bolivia: Univ. Mayor de San Andrés. 157 p.

16. López A, Morales R, Cabrera C, Urra C. 2000. Ingestión y digestibilidad aparente de forrajes por la llama (Lama glama). I. heno de alfalfa (Medicago sativa) y paja de trigo (Triticum aestivum) en diferentes proporciones. Arch Med Vet 32: 201-208. doi: 10.4067/ S0301-732. 
17. Macuchapi D. 2006. Comparación de la suplementación alimenticia al destete con la crianza tradicional de llamas en praderas nativas. Tesis de Ingeniero Agrónomo. La Paz, Bolivia: Univ. Mayor de San Andrés. $73 \mathrm{p}$.

18. Mamani-Linares LW, Cayo F, Gallo C. 2014. Características de canal, calidad de carne y composición química de carne de llama: una revisión. Rev Inv Vet Perú 25: 123-150. doi: 10.15381/ rivep.v25i2.8484

19. Mamani-Linares LW, Gallo CB. 2013a. Meat quality attributes of the Longissimus lumborum muscle of the Kh'ara genotype of llama (Lama glama) reared extensively in northern Chile. Meat Sci 94: 89-94. doi: 10.1016/ j.meatsci.2012.12.013

20. Mamani-Linares LW, Gallo CB. 2013b. Effects of supplementary feeding on carcass and meat quality traits of young llamas (Lama glama). Small Ruminant Res 114: 233-239.

21. Pérez, P, Maino M, Guzman R, Vaquero C, Kobrich, C, Pokniak J. 2000. Carcass characteristics of llamas (Lama glama) reared in central Chile. Small Ruminant Res 37: 93-97. doi: 10.1016/ S0921-4488(99)00127-3
22. Salvá B. 2000. Utilización de proteína de soya y Carragenina en salchichas tipo Huacho con bajo tenor graso. Tesis de Industrias Alimentarias. Lima: Univ. Nacional Agraria La Molina. 164 p.

23. San Martín F. 1996. Nutrición en alpacas y llamas. Publicación Científica $\mathrm{N}^{\circ}$ 27. Lima, Perú:Univ. Nacional Mayor de San Marcos. 29 p.

24. San Martin F, Bryant FC. 1989. Nutrition of domesticated South American llamas and alpacas. Small Ruminant Res: 191-216. doi: 10.1016/09214488(89)90001-1

25. San Martín F, Van Sau NR. 2014. Applied digestive anatomy and feeding behavior. In: Llama and alpaca care. Medicine, surgery, reproduction, nutrition, and herd health. Elsevier. p 51-58.

26. Turín C. 1999. Influencia de la alimentación con pastos naturales y cultivados en alpacas tuis Huacaya de 6 y 18 meses de edad. Tesis de Ingeniero Zootecnista. Lima: Univ. Nacional Agraria La Molina. $110 \mathrm{p}$.

27. Van Saun RJ. 2006. Nutrient requirements of South American camelids: a factorial approach. Small Ruminant Res 61: 165-186. doi: 10.1016/j.smallrumres.2005.07.006 